\title{
Coronal Magnetic Field Structure and Evolution for Flaring AR 11117 and its Surroundings
}

Tilaye Tadesse ${ }^{12}$, T. Wiegelmann ${ }^{1}$, and

B. Inhester ${ }^{1}$, and A. Pevtsov ${ }^{3}$

(c) Springer $\bullet \bullet \bullet \bullet$

\begin{abstract}
In this study, photospheric vector magnetograms obtained with the Synoptic Optical Long-term Investigations of the Sun survey (SOLIS), are used as boundary conditions to model the three-dimensional nonlinear force-free (NLFF) coronal magnetic fields as a sequence of nonlinear force-free equilibria in spherical geometry. We study the coronal magnetic field structure inside active regions and its temporal evolution. We compare the magnetic field configuration obtained from NLFF extrapolation before and after flaring event in active region (AR) 11117 and its surroundings observed on 27 October 2010. We compare the magnetic field topologies and the magnetic energy densities and study the connectivities between AR 11117 and its surroundings. During the investigated time period, we estimate the change in free magnetic energy from before to after the flare to be $1.74 \times 10^{32} \mathrm{erg}$ which represents about $13.5 \%$ of nonlinear forcefree magnetic energy before the flare. In this study, we find that electric currents from AR 11117 to its surroundings were disrupted after the flare.
\end{abstract}

Keywords: Solar flare Magnetic fields Photosphere Corona

\section{Introduction}

The structure of the Sun's corona is dominated by its magnetic field. To understand eruptive events (flares, coronal mass ejections (CMEs) or filament eruptions), we need to follow the evolution of the 3D magnetic configuration (geometry and topology) (Schrijver and Title, 2011). Knowledge of the amount of free magnetic energy and its temporal variation during CMEs/flares will help our quantitative understanding of solar explosive phenomena (Bleybel et al.,

\begin{tabular}{|l|}
\hline 1 Max Planck Institut für Sonnensystemforschung, \\
\hline Max-Planck Str. 2, D-37191 Katlenburg-Lindau, Germany \\
\hline email: tilaye.tadesse@gmail.com email: \\
\hline wiegelmann@mps.mpg.de email: inhester@mps.mpg.de \\
${ }^{2}$ Addis Ababa University, College of Natural Sciences, \\
\hline Institute of Geophysics, Space Science, and Astronomy, \\
\hline Po.Box 1176, Addis Ababa, Ethiopia \\
${ }^{3}$ National Solar Observatory, Sunspot, NM 88349, U.S.A. \\
\hline email: apevtsov@nso.edu \\
\hline
\end{tabular}


2002 Régnier and Canfield, 2006; Thalmann, Wiegelmann, and Raouafi, 2008; Jing et al., 2010). However, routine measurements of the solar magnetic field are mainly carried out in the photosphere. The difficulties of measuring the coronal field and its embedded electrical currents thus leads us to use numerical modelling to infer the field strength in the higher layers of the solar atmosphere from the measured photospheric field.

Nonlinear force-free field (NLFFF) models are thought to be viable tools for investigating the structure, dynamics, and evolution of the coronae of solar active regions. NLFFF models were successfully applied to analytical test cases (Schrijver et al., 2006; Metcalf et al., 2008), but they were less successful in applications to real solar data. Different NLFFF models have been found to yield markedly different field line configurations and widely varying estimates of the magnetic free energy in the coronal volume, when applied to solar data (DeRosa et al., 2009). The main reasons for that problem are (1) the forces acting on the plasma within the photosphere, (2) the uncertainties of vectorfield measurements, particularly of the transverse component, and (3) the large domain that needs to be modelled to capture the connections of an active region to its surroundings. Therefore, nonlinear force-free modeling is not a routine procedure which is guaranteed to produce meaningful results unless the above points are taken into account (DeRosa et al., 2009: Schrijver, 2009). In this study, we have considered those points explicitly. However, caution is still needed when assessing results from this modeling. This is because many aspects of the specific approach to modeling used in this work, such as the use of preprocessed boundary data, the missing boundary data, measurement error due to noise, resolution of a magnetogram and the departure of the model fields from the observed boundary fields may influence the results.

Solar flares are thought to be powered by the magnetic free energy (i.e., the difference between the actual magnetic energy and energy of the equivalent potential field) stored in the corona prior to eruption. The storage of free energy requires a non-potential magnetic field, and is therefore associated with a shear or twist in the coronal field away from the potential, current-free state (Priest and Forbes, 2002, Su et al., 2007, Murray, Bloomfield, and Gallagher, 2011). To date, the pre-cursors to flaring are still not fully understood, although there is evidence that flaring is related to changes in the topology or complexity of an active region's magnetic field.

In this study, we model the coronal magnetic field to determine the sources of flaring activity and the temporal evolution of an active region between preand post-flare stages. Assuming that the evolution of the coronal magnetic field above an active region can be described by successive equilibria, we follow in time the magnetic changes of the 3D nonlinear force-free (nlff) fields reconstructed from two photospheric vector magnetograms taken before and after a flare. We use photospheric vector magnetograms as the boundary conditions to model the three-dimensional coronal magnetic fields in spherical geometry. This enables us to accommodate most of the connectivities within AR 11117 and its surroundings. 


\section{Nonlinear force-free field extrapolation}

Except for during eruptions, the magnetic field in the solar corona evolves slowly as it responds to changes in the surface field, implying that the electromagnetic Lorentz forces in this low- $\beta$ environment are relatively weak and that any electrical currents that exist must be essentially parallel or antiparallel to the magnetic field wherever the field is not negligible. Due to the low value of the plasma $\beta$ (the ratio of gas pressure to magnetic pressure), the solar corona is magnetically dominated (Gary, 2001). To describe the equilibrium structure of the static coronal magnetic field when non-magnetic forces are negligible, the force-free assumption is appropriate:

$$
\begin{gathered}
(\nabla \times \mathbf{B}) \times \mathbf{B}=0 \\
\nabla \cdot \mathbf{B}=0 \\
\mathbf{B}=\mathbf{B}_{\mathrm{obs}} \quad \text { on photosphere, }
\end{gathered}
$$

where $\mathbf{B}$ is the magnetic field and $\mathbf{B}_{\mathrm{obs}}$ is measured vector field on the photosphere. Equation (1) states that the Lorentz force vanishes (as a consequence of $\mathbf{J} \| \mathbf{B}$, where $\mathbf{J}$ is the electric current density) and Equation (2) describes the absence of magnetic monopoles. Based on the above assumption, the coronal magnetic field is modelled with nonlinear force-free field (NLFF) extrapolation (Inhester and Wiegelmann, 2006, Valori, Kliem, and Keppens, 2005, Wiegelmann, 2004 Wheatland, 2004; Wheatland and Régnier, 2009, Wheatland and Leka, 2011; Amari and Aly, 2010).

In this study, we extrapolate the three-dimensional NLFF coronal fields from the photospheric boundary as successive equilibria. Milne-Eddington inverted vector magnetograms, obtained by the the Synoptic Optical Long-term Investigations of the Sun survey (SOLIS)/Vector-SpectroMagnetograph (VSM), are used as the boundary conditions. From a mathematical point of view, appropriate boundary condition for force-free modeling are the vertical magnetic field $B_{n}$ and the vertical current $J_{n}$ prescribed only for one polarity of $B_{n}$ (Amari et al., 1997 Amari, Boulmezaoud, and Mikić, 1999 Amari, Boulmezaoud, and Aly, 2006). A direct use of these boundary conditions is implemented in Grad-Rubin codes (Amari, Boulmezaoud, and Mikić, 1999). Practical computations show, however, that the solutions for $J_{n}$ described in DeRosa et al. (2009) $B_{n}^{+}$and $B_{n}^{-}$can differ significantly for real data containing noise and inconsistencies. Wheatland and Régnier (2009) and Wheatland and Leka (2011) implemented a scheme which uses both of $B_{n}^{+}$and $B_{n}^{-}$solutions together with an error approximation to derive a consistent solution. Using the three components of $B$ as boundary condition requires consistent magnetograms, as outlined in Aly (1989). We use preprocessing and relaxation of the boundary condition to derive these consistent data on the boundary.

For modeling the coronal magnetic field above the active region and its surrounding, we use the variational principle originally proposed by Wheatland, Sturrock, and Roumeliotis (2000) and later improved by Wiegelmann (2004) 
in cartesian co-ordinates. The method minimizes a joint measure $\left(\mathcal{L}_{\omega}\right)$ for the normalized Lorentz forces (Equation 1) and the divergence of the field (Equation 2 (each of which should equal zero) throughout the volume of interest, $V$. We have implemented this method for the function $\left(\mathcal{L}_{\omega}\right)$ in spherical geometry (Wiegelmann, 2007) Tadesse, Wiegelmann, and Inhester, 2009) and iterate B to minimize $\mathcal{L}_{\omega}$. The modification concerns the input bottom boundary field $\mathbf{B}_{\mathrm{obs}}$ which the model field $\mathbf{B}$ is not forced to match exactly but we allow deviations of the order of the observational errors. The modified variational problem is (Wiegelmann and Inhester, 2010, Tadesse et al., 2011):

$$
\begin{gathered}
\mathbf{B}=\operatorname{argmin}\left(\mathcal{L}_{\omega}\right), \\
\mathcal{L}_{\omega}=\mathcal{L}_{\mathrm{f}}+\mathcal{L}_{\mathrm{d}}+\nu \mathcal{L}_{\text {photo }} \\
\mathcal{L}_{\mathrm{f}}=\int_{V} \omega_{\mathrm{f}}(r, \theta, \phi) B^{-2}|(\nabla \times \mathbf{B}) \times \mathbf{B}|^{2} r^{2} \sin \theta d r d \theta d \phi \\
\mathcal{L}_{\mathrm{d}}=\int_{V} \omega_{\mathrm{d}}(r, \theta, \phi)|\nabla \cdot \mathbf{B}|^{2} r^{2} \sin \theta d r d \theta d \phi \\
\mathcal{L}_{\text {photo }}=\int_{S}\left(\mathbf{B}-\mathbf{B}_{\mathrm{obs}}\right) \cdot \mathbf{W}(\theta, \phi) \cdot\left(\mathbf{B}-\mathbf{B}_{\mathrm{obs}}\right) r^{2} \sin \theta d \theta d \phi
\end{gathered}
$$

where $\mathcal{L}_{\mathrm{f}}$ and $\mathcal{L}_{\mathrm{d}}$ measure how well the force-free Equations 11 and divergencefree (2) conditions are fulfilled, respectively. $\omega_{\mathrm{f}}(r, \theta, \phi)$ and $\omega_{\mathrm{d}}(r, \theta, \phi)$ are weighting functions for the force-free and divergence-free terms, respectively, and are chosen to be identical for this study. The weighting functions are chosen to be constant within the inner physical domain $V^{\prime}$ and decline to 0 with a cosine profile in the buffer boundary region (see Tadesse, Wiegelmann, and Inhester, Tadesse et al. (2009, 2011)). The third integral, $\mathcal{L}_{\text {photo, }}$ is a surface integral over the photosphere which forces to relax the field $\mathbf{B}$ at the photosphere towards the measured surface field data, $\mathbf{B}_{\mathrm{obs}}$. In this integral, $\mathbf{W}(\theta, \phi)=$ $\operatorname{diag}\left(w_{\text {radial }}, w_{\text {trans }}, w_{\text {trans }}\right)$ is a diagonal matrix which gives different weights to the observed surface field components depending on the relative accuracy in measurement. In this sense, lacking data is considered most inaccurate and is taken account of by setting $W(\theta, \phi)$ to zero in all elements of the matrix. SOLIS/VSM provides full-disk vector-magnetograms, but for some individual pixels the inversion from line profiles to field values may not have been successful and field data there remains undetermined at these pixels. Typically, the field is missing where its magnitude is small so that these pixels have a small impact on the model even if they were measured correctly. Within the error margin of a measured field value, any value is just as good as any other and from this range of values we take the value that fits the force-free field best. The different errors for the radial and transverse components of $\mathbf{B}_{\mathrm{obs}}$ are taken into account by different values for $w_{\text {radial }}$ and $w_{\text {trans }}$. In this work we used $w_{\text {radial }}=20 w_{\text {trans }}$ for the surface preprocessed fields as the radial component of $\mathbf{B}_{\mathrm{obs}}$ is measured with higher accuracy. Figure 1 shows the difference in gauss between the measured 
vector magnetograms and the final values of the model. Hence the final model field values on the boundary are consistent with the observed data within noise levels(noise due to fluctuations in intensity) which are about $1 \mathrm{G}$ and $50 \mathrm{G}$ for longitudinal and transverse components, respectively.

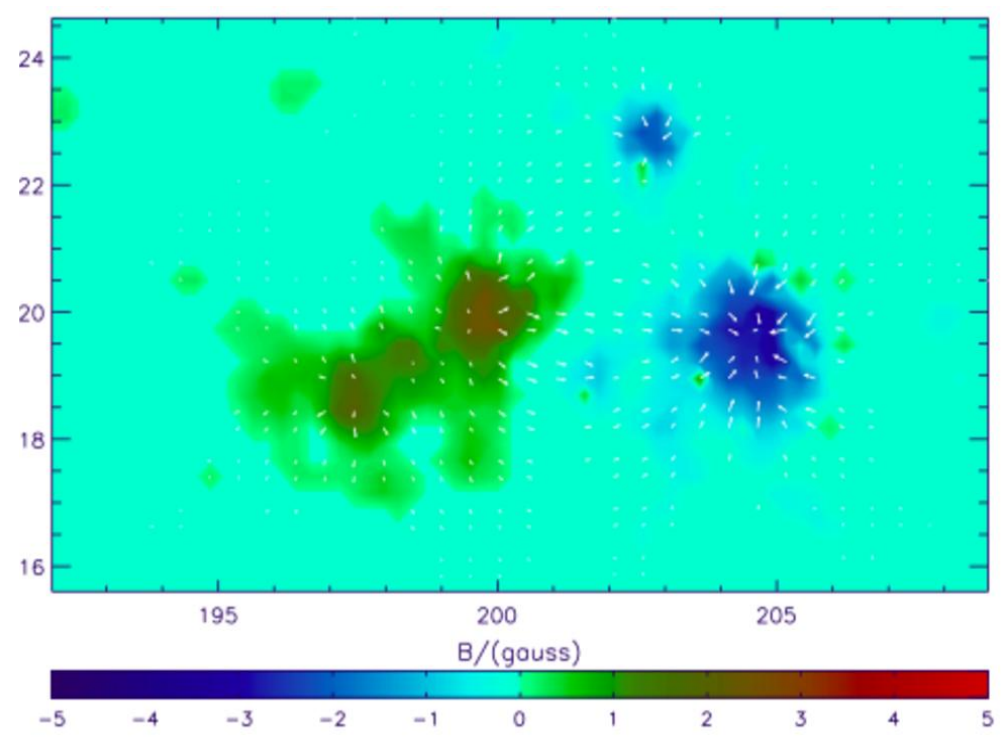

Figure 1. Surface contour plot of radial magnetic field component and vector field plot of transverse field( white arrows) of the difference between original data before preprocessing and the final field values after relaxation through the term $\mathcal{L}_{\text {photo }}$ in Equation (4). The maximum change in the transverse field is $30 \mathrm{G}$ which corresponds to the length of the longest white arrow. The vertical and horizontal axes show latitude, $\theta$ (in degree) and longitude, $\phi$ (in degree) on the photosphere. In the area coloured in light blue, field values are lacking.

We use a spherical grid $r, \theta, \phi$ with $n_{r}, n_{\theta}, n_{\phi}$ grid points in the direction of radius, latitude, and longitude, respectively. For details of the method, we direct the reader to Tadesse et al. (2012). In the present work, we use a larger computational domain which accommodates most of the connectivity within AR 11117 and its surroundings. We also take the uncertainties of measurements in vector magnetograms into account as suggested in DeRosa et al. (2009).

\section{Results}

Solar activity on 27 October 2010 was dominated by NOAA AR 11117. A GOES $\mathrm{C} 1.2$ flare has been observed at $\mathrm{N} 18^{0} \mathrm{~S} 25^{0}$ in the active region. NOAA records indicate that the event began in soft X-rays (SXRs) which were detected by the GOES 15 satellite at 16:59 UT, reaching a peak at 17:04 UT and ending at 17:08 UT (see Figure 2). There were two SOLIS/VSM vector magnetograms 


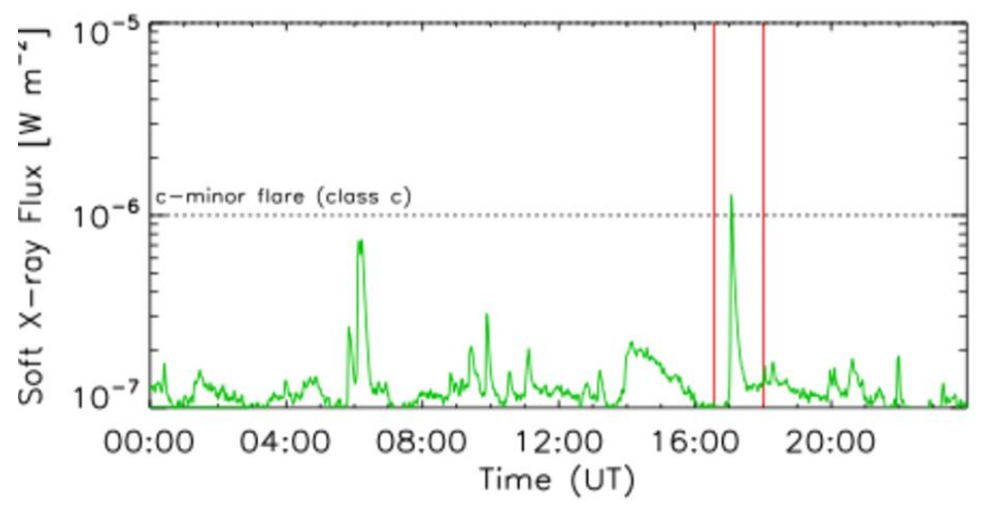

Figure 2. Solar SXR flux on 27 October 2010 in the wavelength range of $0.1-0.8 \mathrm{~nm}$. Blue vertical lines indicate the availability of SOLIS/VSM data.

taken, one about half an hour before and the other about one hour after the flare. In Figure 2, the time the magnetograms were taken is marked by red vertical lines at 16:33 UT before the flare and the other at 18:00 UT after the flare. As a first step, we remove the net forces and torques from the boundary using our spherical preprocessing procedure(Tadesse, Wiegelmann, and Inhester, 2009) which brings the photospheric magnetic field closer to the boundary values of a force-free field (Molodensky, 1969; Aly, 1989). Then we apply our spherical extrapolation scheme using the surface vector field solution obtained from the preprocessing scheme. To determine the 3D coronal magnetic field as a nlff equilibrium, we need the three components of the magnetic field of AR 11117 and its surroundings on the photosphere. The computations are performed in a wedge-shaped computational box of volume $V$ with $140 \times 115 \times 250$ pixels in radial, latitudinal and longitudinal directions. The computational box is large enough to include the connectivity between the AR 11117 and its surroundings. Figure 3 shows the temporal variations of the photospheric vector magnetic components of AR 11117 before and after the flare. The normal component is color coded and the horizontal components are shown as surface vectors. In order to quantify the change in the surface vector magnetic field, we computed the vector correlation between the fields before and after the flare. We use the vector correlation $\left(C_{\mathrm{vec}}\right)$ (Schrijver et al., 2006) metric which generalizes the standard correlation coefficient for scalar functions and is given by

$$
C_{\mathrm{vec}}=\frac{\sum_{i} \mathbf{v}_{i} \cdot \mathbf{u}_{i}}{\sqrt{\sum_{i}\left|\mathbf{v}_{i}\right|^{2}} \sqrt{\sum_{i}\left|\mathbf{u}_{i}\right|^{2}}}
$$




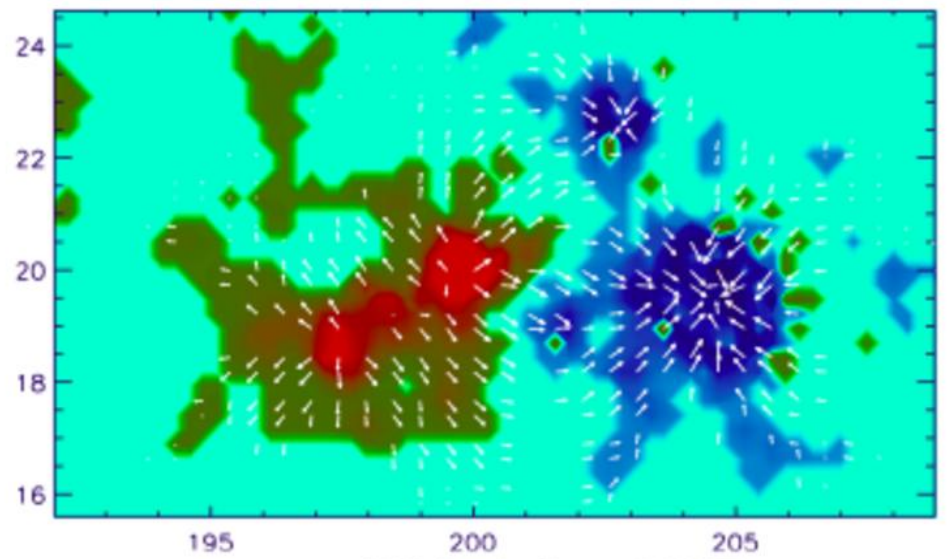

(a) Before the flare at 16:33 UT

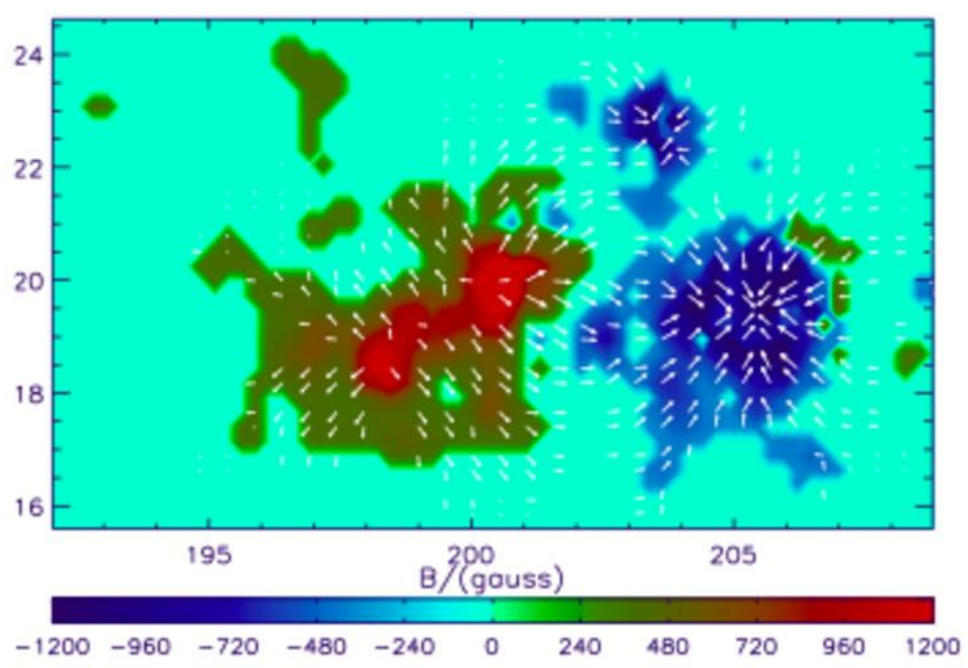

(b) After the flare at 18:00 UT

Figure 3. Surface contour plot of radial magnetic field component and vector field plot of transverse field with white arrows. The color coding shows $B_{r}$ on the photosphere. The vertical and horizontal axes show latitude, $\theta$ (in degree) and longitude, $\phi$ (in degree) on the photosphere. In the area coloured in light blue, field values are lacking. 
where $\mathbf{v}_{i}$ and $\mathbf{u}_{i}$ are $2 \mathrm{D}$ vectors at grid point $i$. If the vector fields are identical, then $C_{\mathrm{vec}}=1$; if $\mathbf{v}_{i} \perp \mathbf{u}_{i}$, then $C_{\mathrm{vec}}=0$. The correlation $\left(C_{\mathrm{vec}}\right)$ of the $2 \mathrm{D}$ surface magnetic field vectors before and after the flare are 0.96 and 0.87 for the radial and transverse components, respectively. From these values we can see that there has been some change in the surface magnetic field configuration during the flare event. The change in the surface magnetic field is towards an intensification in transverse components. This change in transverse components indicates that there is also the change in the vertical components of electric current density. Figure 4 shows the temporal variation of the vertical electric current density on the photosphere. We computed the vertical electric current density on the surface using the relation $J_{r}=\hat{\mathbf{r}} \cdot \nabla \times \mathbf{B}$ (where $\mathbf{B}$ the magnetic field) from the transverse magnetic field components. We depict a surface color plot of the vertical electric current densities before and after the flare in Figures 4 a and $\mathrm{b}$, respectively. In order to quantify the change in the radial electric current densities on the photosphere, we calculate the total of the absolute value of the vertical electric current density before and after flare. We use the pointwise sum of the values at nodal points. We find a ratio of the total absolute value of the vertical electric current density after the flare to that before the flare to be 0.863 . The vertical electric current in the active region therefore has decreased after the flare.

Magnetic fields are generally recognized as playing a fundamental role in flares. During the course of a flare, the magnetic field is believed to undergo major changes (Jing et al., 2009). In this work we have compared the magnetic field topologies from two datasets taken before and after the flare using our $3 \mathrm{D}$ NLFF reconstruction method. From a visual inspection of the magnetic field lines within the extrapolation volume, we recognize some changes in the magnetic field structure during the C1.2 flare. Figure 5 shows some selected magnetic field lines from our reconstructions before and after the flare along with the respective SDO/AIA images. Figure 6 shows the magnetic field lines of Figure 5 zoomed in and rotated to the limb. In order to compare the fields at the two consecutive datasets quantitatively, we computed the vector correlations between the $3 \mathrm{D}$ field configurations. The correlations $\left(C_{\mathrm{vec}}\right)$ of the $3 \mathrm{D}$ magnetic field vectors before and after the flare with respect to the potential field configuration before the flare are 0.81 and 0.93 respectively. We see that the magnetic field configuration after the flare looses some of its non-potentiality.

The energy stored in the magnetic field as a result of fieldline stressing into a non-potential configuration has been identified as the source of flare energy. The study by Jing et al. (2010) confirms that there is physical link between magnetic energy and flare occurrence in active regions. Study of the temporal evolution of the free magnetic energy indicates that it varies before and after the flare events (Jing et al., 2009). There is strong need to estimate this free energy numerically. One way to estimate the energy budget of active regions is to reconstruct the three-dimensional (3D) coronal field from the measured photospheric boundary based on the force-free assumption. We compute the free magnetic energy from the excess energy of the extrapolated field beyond that of the potential field which satisfies the same $\mathbf{B}_{\mathrm{obs}} \cdot \hat{r}$ boundary condition. Similar estimates have been made by Régnier and Priest (2007), Thalmann, Wiegelmann, and Raouafi (2008), 


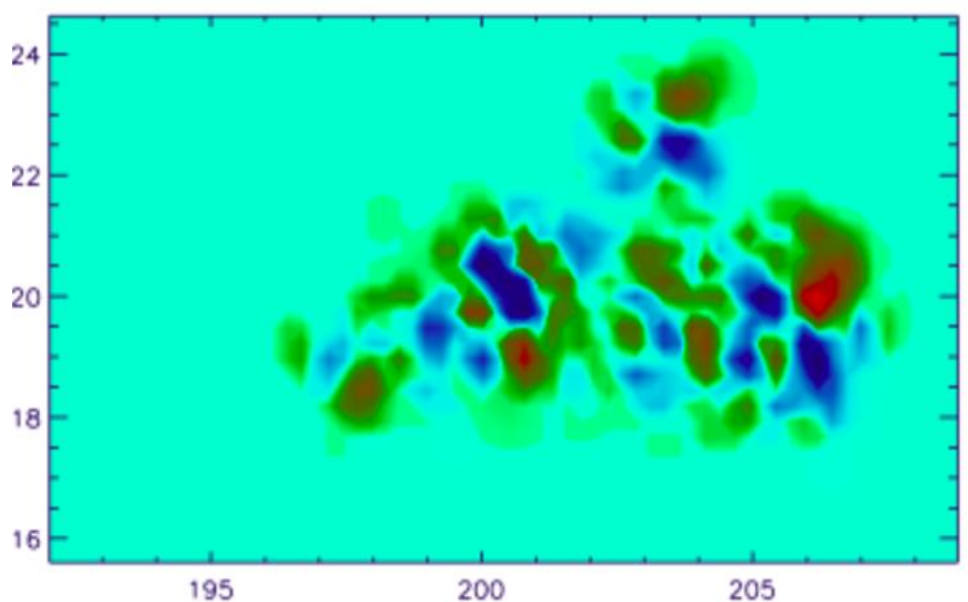

(a) Before the flare at 16:33 UT

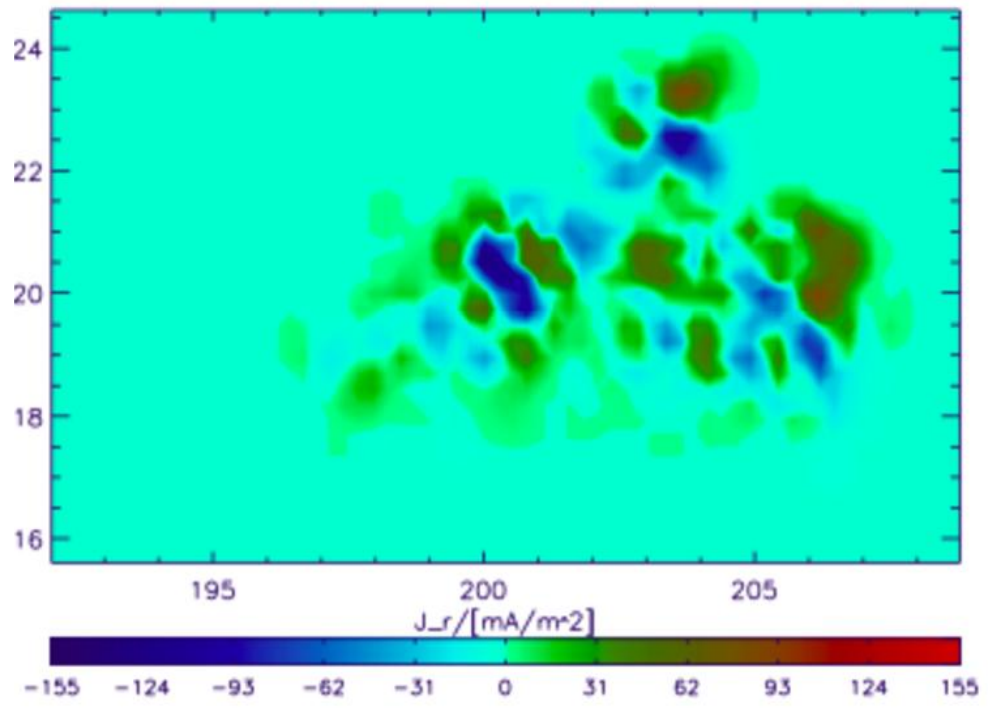

(b) After the flare at 18:00 UT

Figure 4. Surface contour plot of the radial component of electric current density. The color coding shows $J_{r}$ on the photosphere. The vertical and horizontal axes show latitude, $\theta$ (in degree) and longitude, $\phi$ (in degree) on the photosphere. In the area coloured in light blue, field values are lacking. 


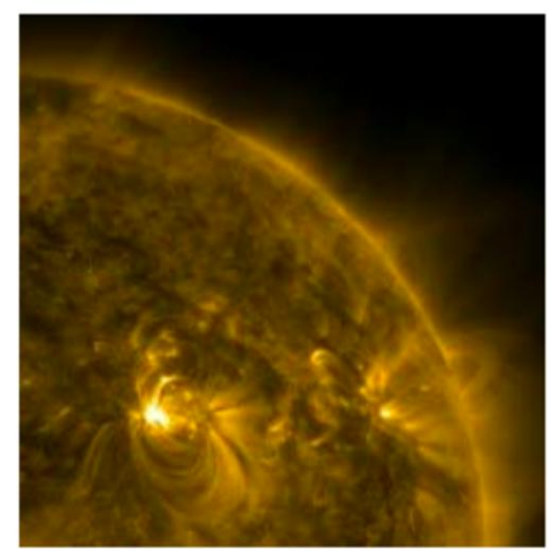

(a) AIA image at 16:35 UT

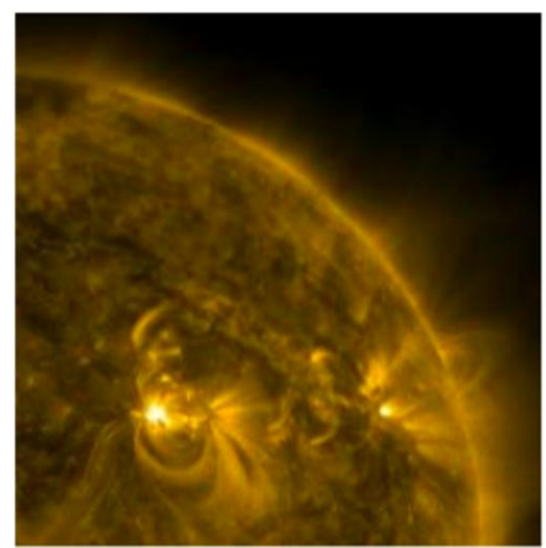

(c) AIA image at 18:05 UT

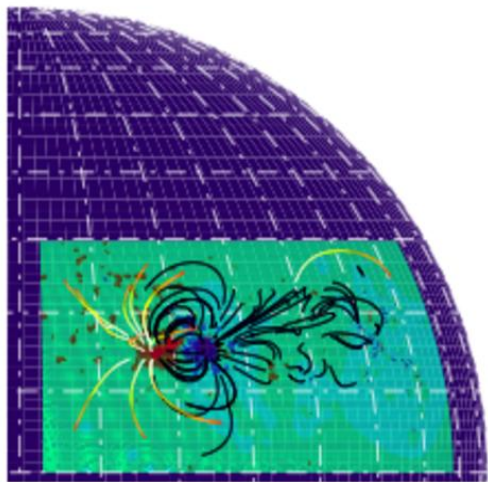

(b) NLFF solution at 16:33 UT

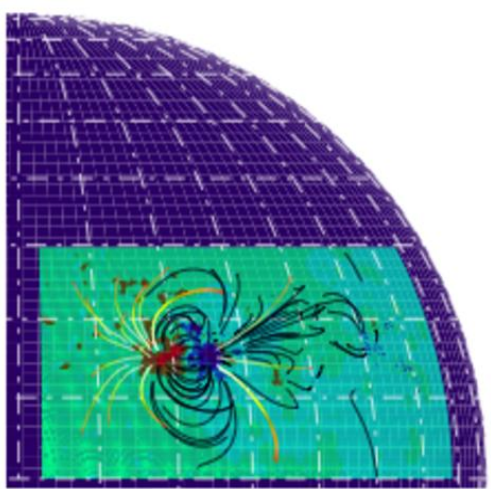

(d) NLFF solution at 18:00 UT

Figure 5. SDO/AIA ( textitSolar Dynamics Observatory/ Atmospheric Imaging Assembly) $171 \AA$ images and their respective selected magnetic field lines reconstructed from SOLIS magnetograms using nonlinear force-free modelling.

and Tadesse et al. (2012) for active regions observed at other times. From the corresponding potential and force-free magnetic field, $\mathbf{B}_{\text {pot }}$ and $\mathbf{B}$, respectively, we can estimate an upper limit to the free magnetic energy associated with coronal currents

$$
E_{\text {free }}=E_{\text {nlff }}-E_{\text {pot }}=\frac{1}{8 \pi} \int_{V^{\prime}}\left(B_{\mathrm{nlff}}^{2}-B_{\text {pot }}^{2}\right) r^{2} \sin \theta d r d \theta d \phi .
$$

The computed energy values are listed in Table 1. The change in the free energy during the flare is about $1.74 \times 10^{32} \mathrm{erg}$. The magnetic energy of the potential 


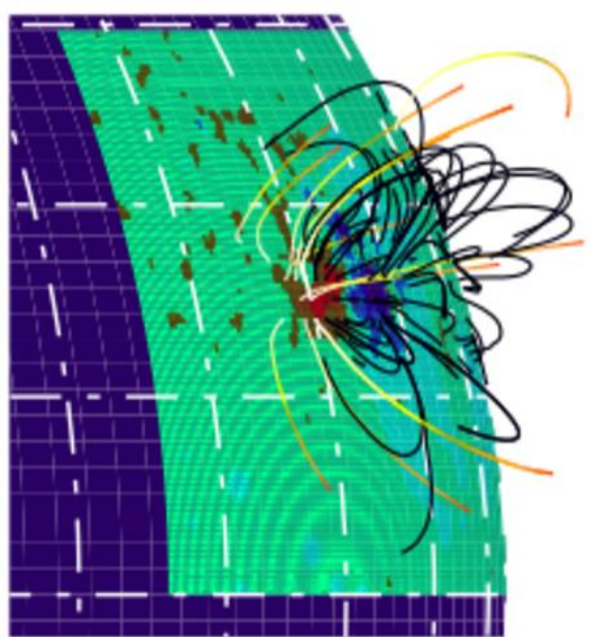

(a) NLFF solution before the flare at 16:33 UT

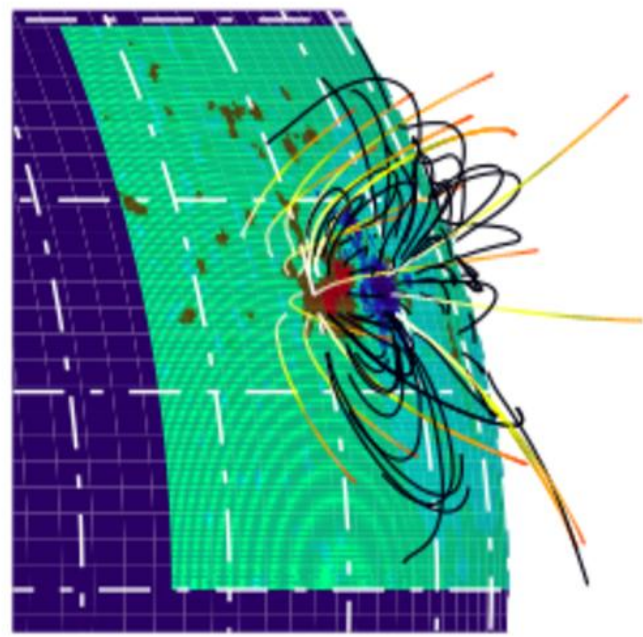

(b) NLFF solution after the flare at 18:00 UT

Figure 6. Some magnetic field lines reconstructed from SOLIS magnetograms. These Figures are obtained by zooming and rotating Figures $5 \mathrm{p}$ and $\mathrm{d}$ to the solar limb. 
Table 1. The magnetic energy obtained from potential and NLFF field extrapolations before and after the flare within the computational box.

\begin{tabular}{cccc}
\hline Events & $E_{\text {nlff }}\left(10^{32} \mathrm{erg}\right)$ & $E_{\text {pot }}\left(10^{32} \mathrm{erg}\right)$ & $E_{\text {free }}\left(10^{32} \mathrm{erg}\right)$ \\
\hline Before the flare at 16:33UT & 12.89 & 10.84 & 2.05 \\
After the flare at 18:00UT & 10.93 & 10.62 & 0.31 \\
\hline
\end{tabular}

field configuration is about $10 \times 10^{32} \mathrm{erg}$. $E_{\text {nlff }}$ exceeds $E_{\text {pot }}$ by only $15.9 \%$ and $2.8 \%$ for before and after the flare, respectively. To estimate the uncertainty in the numerical result, the code was applied to the original SOLIS data to which artificial random noise had been added in the form of a normal distribution with an amplitude of approximately $1 \mathrm{G}$ in the longitudinal and $50 \mathrm{G}$ in the transverse component. The chosen noise amplitudes are based on the sensitivity of the VSM instrument. Hence, we found that the evaluated relative error of the energy estimation is about $0.4 \%$ for the potential and $1 \%$ for the NLFF field (i.e. $E_{\text {pot }} \pm 0.044 \times 10^{32} \mathrm{erg}$ and $E_{\text {nlff }} \pm 0.129 \times 10^{32} \mathrm{erg}$, respectively). The available free magnetic energy is approximately $10^{32} \mathrm{erg}$ with a relative error of about $4 \%$ (i.e. $E_{\text {free }} \pm 0.082 \times 10^{32} \mathrm{erg}$ ). In principle high spatial resolution of a magnetogram gives a higher magnetic energy because small-scale magnetic variations are better resolved. How much better resolution contributes to the total energy estimate depends on the steepness of the spectral energy distribution. Steeper spectra yield less additional energy if the resolution is enhanced, flatter spectra yield more additional energy. In addition to our relative error estimates above, low spatial resolution might increase the error.

To study the influence of the use of preprocessed boundary data along with the departure of the model field from the observed boundary fields on the estimation of free-magnetic energy, we have computed the magnetic energy of the potential field and the NLFFF obtained from the original data without preprocessing and with preprocessing. As the preprocessing procedure filters out small-scale surface field fluctuations, the magnetic energy of NLFFF obtained from preprocessed boundary data is smaller than the corresponding energy without preprocessing. The energy of the potential field obtained from boundary data with and without preprocessing are close in value, since the potential field calculation makes only the use of the radial magnetic field component which is not affected too much by the preprocessing. The magnetic energy computed from the original data without preprocessing is about $13.67 \times 10^{32} \mathrm{erg}$ which is about $6 \%$ higher than the one obtained from preprocessed and modified observational boundary data. However, this energy does not correspond to the nonlinear force-free magnetic field solution since the original boundary data without preprocessing is not a consistent boundary condition for NLFFF modeling.

In our previous work (Tadesse et al., 2012), we have studied the connectivity between three neighbouring active regions. In that study, we investigated the three ARs were found to share a significant amount of magnetic flux compared to their internal flux connecting one polarity to the other. In terms of the electric current they were much more isolated. In this work, we study the connectivity 


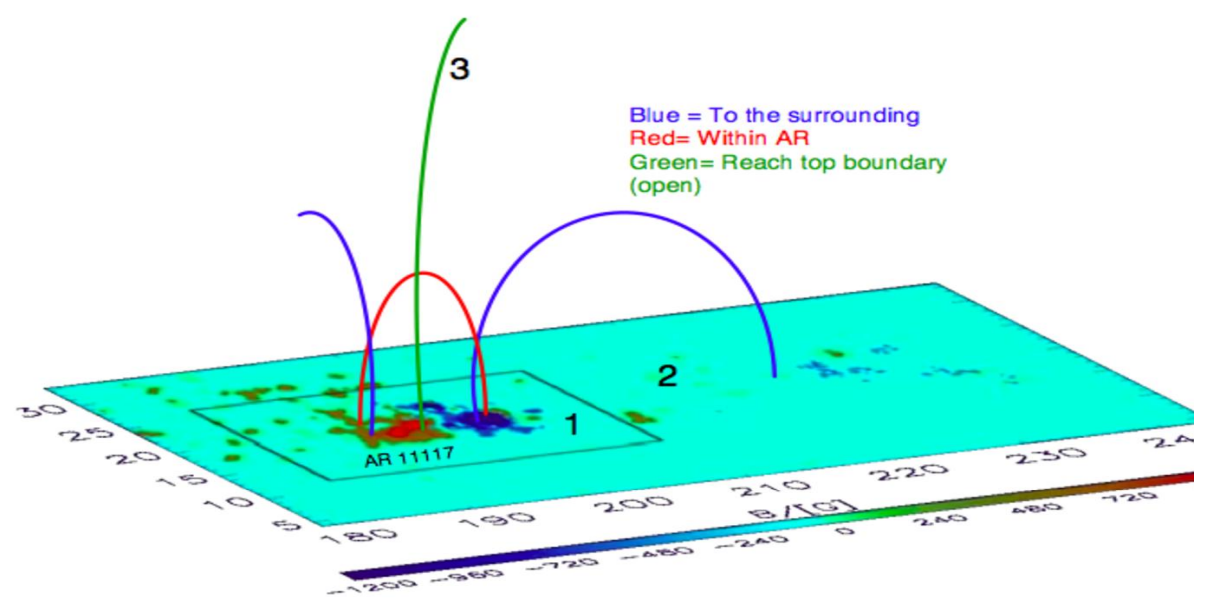

Figure 7. The connectivity between AR 11117 and its surroundings. The black rectangle shows the domain of AR 11117. The color coding shows $B_{r}$ on the photosphere. The red fieldline represents fieldlines connecting opposite polarities within the active region. The blue one represents fieldlines connecting the active region with its surroundings both on the photosphere and the side boundaries (categorized as elsewhere). The green one shows fieldlines, which leave the computational box though its top boundary and may be considered to be "open".

between AR 11117 and its surroundings before and after flare. In order to quantify these connectivities, we have calculated the magnetic flux and the electric currents shared between the active region and its surroundings. For the magnetic flux, e.g., we use

$$
\Phi_{\alpha \beta}=\sum_{i}\left|\mathbf{B}_{i} \cdot \hat{r}\right| R_{\odot}^{2} \sin \left(\theta_{i}\right) \Delta \theta_{i} \Delta \phi_{i}
$$

where the summation is over all pixels of $\mathrm{AR}_{\alpha}$ from which the field line ends in $\mathrm{AR}_{\beta}$ or $i \in \mathrm{AR}_{\alpha} \|$ conjugate footpoint $(i) \in \mathrm{AR}_{\beta}$. The indices $\alpha$ and $\beta$ may take values between 1 and 3 . The index number 1 corresponds to AR 11117, index number 2 to its surroundings and the side boundaries and index number 3 to the top boundary (see Figure 7). For the electric current we replace the magnetic field, $\mathbf{B}_{i} \cdot \hat{r}$, by the vertical current density $\mathbf{J}_{i} \cdot \hat{r}$ in Equation (7). Both Table 2 and 3 show the percentage of the total magnetic flux and electric current shared between the AR 11117 and its surroundings before and after the flaring event. For example, first column of Table 2 shows that $82.23 \%$ of positive polarity of AR 11117 is connected to negative polarity within itself; line 2 shows that $41.51 \%$ of positive/negative polarity of AR 11117 is connected to positive/negative polarity of its surroundings including the side boundaries of the computational box, and line 3 shows that $74.78 \%$ of the total magnetic flux of top boundary of the computational box connected to the positive/negative polarity of AR 11117 . Table 3 shows the electric current connectivity we have calculated applying the same technique. Figure 4 shows that the vertical electric current density has 
Table 2. The percentage of the total magnetic flux shared between AR 11117 and its surroundings. $\Phi_{11}, \Phi_{22}$ and $\Phi_{33}$ denote magnetic flux of AR 11117 , outside AR 11117 on the photosphere including the side boundaries and the top boundary of the computational box (see Figure 7), respectively.

\begin{tabular}{rlccccr} 
& \multicolumn{3}{c}{ Before the flare } & \multicolumn{3}{c}{ After the flare } \\
\cline { 2 - 7 }$\Phi_{\alpha \beta}$ & $\beta=1$ & 2 & 3 & $\beta=1$ & 2 & 3 \\
\hline$\alpha=1$ & 82.23 & 12.61 & 5.16 & 88.37 & 7.36 & 4.27 \\
2 & 41.51 & 50.94 & 7.55 & 35.18 & 56.24 & 8.58 \\
3 & 74.78 & 25.22 & 0.00 & 65.49 & 34.51 & 0.00 \\
\hline
\end{tabular}

Table 3. The percentage of the total electric current shared between AR 11117 and its surroundings. $I_{11}, I_{22}$, and $I_{33}$ denote electric current within AR 11117, outside AR 11117 on the photosphere including the side boundaries and the top boundary of the computational box (see Figure 7), respectively.

\begin{tabular}{rlccrcr} 
& \multicolumn{3}{c}{ Before the flare } & \multicolumn{3}{c}{ After the flare } \\
\cline { 2 - 7 }$I_{\alpha \beta}$ & $\beta=1$ & 2 & 3 & $\beta=1$ & 2 & 3 \\
\hline$\alpha=1$ & 93.02 & 6.98 & 0.00 & 98.06 & 1.94 & 0.00 \\
2 & 34.41 & 65.59 & 0.00 & 26.38 & 73.62 & 0.00 \\
3 & 0.00 & 0.00 & 0.00 & 0.00 & 0.00 & 0.00 \\
\hline
\end{tabular}

decreased after the flare. In this study (see Table 3), we found that AR 11117 is even more isolated in electric current from its surroundings after the flare. It is noteworthy that modeling this active region with small cartesian box would lead to wrong NLFF model solution as there are currents crossing its boundaries.

\section{Conclusions}

We have investigated the coronal magnetic field associated with AR 11117 and its surroundings observed on 27 October 2010 by analysing SOLIS/VSM data. Two vector magnetograms with a time cadence of 1 hour and 28 min were available to investigate the magnetic energy content of the coronal field during the C1.2 flare observed by GOES. We have used an optimization method for the reconstruction of nonlinear force-free coronal magnetic fields in spherical geometry by restricting the code to limited parts of the Sun (Wiegelmann, 2007; Tadesse, Wiegelmann, and Inhester, 2009; Tadesse et al., 2011; Tadesse et al., 2012).

We have studied the time evolution of the magnetic field from before to after the flare. We found that there is some rearrangement in the magnetic field configuration after the eruption. The magnetic energies calculated in a large wedge-shaped computational box above the active region and its surroundings decreased after the flare, indicating that the field looses some of its non-potentiality. However, caution is needed when estimating the free magnetic energy using NLFFF modeling. Many aspects of the specific approach used in NLFFF modeling may influence the results. This is the first study which contains AR and its surroundings with a flaring event in our model. It was made possible by the use of spherical coordinates and allowed us to analyse connectivity between AR and its surroundings. Modeling an active region in cartesian geometry 
would lead to wrong NLFF model solution as there are currents crossing the small cartesian box enclosing it by excluding its surroundings. We propose to systematically study the effect of using cartesian box over spherical wedge-shaped box for NLFF solutions.

High cadence magnetogram observations are necessary when we study the magnetic field topology and energy variations associated with CME/flare eruptions. In this sense, it is worth mentioning that the Helioseismic and Magnetic Imager (HMI) on board the Solar Dynamic Observatory (SDO) is the first instrument to provide routine measurements of the full-disk photospheric vector magnetogram data with high spatial and temporal resolution under seeing-free condition. We anticipate extending the current study with soon to be released SDO/HMI full-disk vector magnetograms.

\section{Acknowledgements}

SOLIS/VSM vector magnetograms are produced cooperatively by NSF/NSO and NASA/LWS. The National Solar Observatory (NSO) is operated by the Association of Universities for Research in Astronomy, Inc., under cooperative agreement with the National Science Foundation. Tilaye Tadesse Asfaw acknowledges a fellowship of the International Max-Planck Research School at the Max-Planck Institute for Solar System Research and the work of T. Wiegelmann was supported by DLR-grant 50 OC 0501.

\section{References}

Aly, J.J.: 1989, Solar Phys. 120, 19.

Amari, T., Aly, J.: 2010, Astron. Astrophys. 522, A52.

Amari, T., Boulmezaoud, T.Z., Aly, J.J.: 2006, Astron. Astrophys. 446, 691.

Amari, T., Boulmezaoud, T.Z., Mikić, Z.: 1999, Astron. Astrophys. 350, 1051.

Amari, T., Aly, J.J., Luciani, J.F., Boulmezaoud, T.Z., Mikic, Z.: 1997, Solar Phys. 174, 129.

Bleybel, A., Amari, T., van Driel-Gesztelyi, L., Leka, K.D.: 2002, Astron. Astrophys. 395, 685.

DeRosa, M.L., Schrijver, C.J., Barnes, G., Leka, K.D., Lites, B.W., Aschwanden, M.J., et al.: 2009, Astrophys. J. 696, 1780.

Gary, G.A.: 2001, Solar Phys. 203, 71.

Inhester, B., Wiegelmann, T.: 2006, Solar Phys. 235, 201.

Jing, J., Chen, P.F., Wiegelmann, T., Xu, Y., Park, S.H., Wang, H.: 2009, Astrophys. J. 696, 84.

Jing, J., Tan, C., Yuan, Y., Wang, B., Wiegelmann, T., Xu, Y., Wang, H.: 2010, Astrophys. J. 713, 440 .

Metcalf, T.R., De Rosa, M.L., Schrijver, C.J., Barnes, G., van Ballegooijen, A.A., Wiegelmann, T., Wheatland, M.S., Valori, G., McTtiernan, J.M.: 2008, Solar Phys. 247, 269.

Molodensky, M.M.: 1969, Soviet Astron. 12, 585.

Murray, S.A., Bloomfield, D.S., Gallagher, P.T.: 2012, Solar Phys. 277, 45.

Priest, E.R., Forbes, T.G.: 2002, Astron. Astrophys. Rev. 10, 313.

Régnier, S., Canfield, R.C.: 2006, Astron. Astrophys. 451, 319.

Régnier, S., Priest, E.R.: 2007, Astrophys. J. Lett. 669, L53.

Schrijver, C.J.: 2009, Adv. Space Res. 43, 739.

Schrijver, C.J., Title, A.M.: 2011, J. Geophys. Res. 116, A04108.

Schrijver, C.J., Derosa, M.L., Metcalf, T.R., Liu, Y., McTiernan, J., Régnier, S., Valori, G., Wheatland, M.S., Wiegelmann, T.: 2006, Solar Phys. 235, 161. 
Su, Y., Golub, L., van Ballegooijen, A., Deluca, E.E., Reeves, K.K., Sakao, T., Kano, R., Narukage, N., Shibasaki K.: 2007, Publ. Astron. Soc. Japan 59, 785.

Tadesse, T., Wiegelmann, T., Inhester, B.: 2009, Astron. Astrophys. 508, 421.

Tadesse, T., Wiegelmann, T., Inhester, B., Pevtsov, A.: 2011, Astron. Astrophys. 527, A30.

Tadesse, T., Wiegelmann, T., Inhester, B., Pevtsov, A.: 2012, Solar Phys. 277, 119.

Thalmann, J.K., Wiegelmann, T., Raouafi, N.E.: 2008, Astron. Astrophys. 488, L71.

Valori, G., Kliem, B., Keppens, R.: 2005, Astron. Astrophys. 433, 335.

Wheatland, M.S.: 2004, Solar Phys. 222, 247.

Wheatland, M.S., Leka, K.D.: 2011, Astrophys. J. 728, 112.

Wheatland, M.S., Régnier, S.: 2009, Astrophys. J. Lett. 700, L88.

Wheatland, M.S., Sturrock, P.A., Roumeliotis, G.: 2000, Astrophys. J. 540, 1150.

Wiegelmann, T.: 2004, Solar Phys. 219, 87.

Wiegelmann, T.: 2007, Solar Phys. 240, 227.

Wiegelmann, T., Inhester, B.: 2010, Astron. Astrophys. 516, A107. 